\title{
Role of HERG1 potassium channel in both malignant transformation and disease progression in head and neck carcinomas
}

\author{
Sofía T Menéndez*,1, Juan P Rodrigo*,1, Saúl Álvarez-Teijeiro ${ }^{1}$, M Ángeles Villaronga ${ }^{1}$, \\ Eva Allonca ${ }^{1}$, Aitana Vallina ${ }^{2}$, Aurora Astudillo ${ }^{2}$, Francisco Barros ${ }^{3}$, Carlos Suárez ${ }^{1}$ and \\ Juana M García-Pedrero ${ }^{1}$ \\ ${ }^{1}$ Servicio de Otorrinolaringología, Instituto Universitario de Oncología del Principado de Asturias, Hospital \\ Universitario Central de Asturias, Oviedo, Spain; ${ }^{2}$ Servicio de Anatomía Patológica, Instituto Universitario \\ de Oncología del Principado de Asturias, Hospital Universitario Central de Asturias, Oviedo, Spain and \\ ${ }^{3}$ Departamento de Bioquímica y Biología Molecular, Universidad de Oviedo, Oviedo, Spain
}

\begin{abstract}
Evidence indicates that human ether à-go-go-related gene 1 (HERG1) voltage-gated potassium channels could represent new valuable membrane therapeutic targets and diagnostic/prognostic biomarkers in various cancers. This study is the first to investigate the expression pattern of HERG1 potassium channel subunit in both primary tumors and precancerous lesions to establish its clinical and biological role during the development and progression of head and neck squamous cell carcinomas. HERG1 protein expression was evaluated by immunohistochemistry in paraffin-embedded tissue specimens from 133 patients with laryngeal/ hypopharyngeal squamous cell carcinomas and 75 patients with laryngeal dysplasia, and correlated with clinical data. Our findings demonstrate that HERG1 is frequently aberrantly expressed in a high percentage of primary tumors $(87 \%)$, whereas expression was negligible in both stromal cells and normal-adjacent epithelia. HERG1 expression increased during head and neck squamous cell carcinoma progression and was significantly associated with lymph node metastasis $(P=0.04)$, advanced disease stages $(P<0.001)$, regional tumor recurrence $(P=0.004)$, distant metastasis $(P=0.03)$ and reduced disease-specific survival $(P=0.012$, logrank test). HERG1-positive expression was also detected in 31 (41\%) of 75 laryngeal dysplasias. Interestingly, HERG1 expression increased with the grade of dysplasia; however, HERG1 expression but not histology correlated significantly with increased laryngeal cancer risk $(P=0.007)$. In addition, functional studies in head and neck squamous cell carcinoma-derived cell lines further revealed that HERG1 expression promotes anchorage-dependent and -independent cell growth and invasive capability, although independently of its ionconducting function. Our data demonstrate that HERG1 expression is a biologically and clinically relevant feature in head and neck squamous cell carcinoma progression and also during malignant transformation, and a promising candidate as cancer risk marker and therapeutic target for head and neck squamous cell carcinoma prevention and treatment.

Modern Pathology (2012) 25, 1069-1078; doi:10.1038/modpathol.2012.63; published online 30 March 2012
\end{abstract}

Keywords: cancer risk; dysplasia; head and neck squamous cell carcinoma; HERG1; immunohistochemistry; metastasis; potassium channel

The incidence of head and neck squamous cell carcinoma has been gradually increasing over the

Correspondence: Dr JM García-Pedrero, PhD, Laboratorio 2 ORL - IUOPA, Hospital Universitario Central de Asturias, 33006 Oviedo, Spain.

E-mail: juanagp@ficyt.es

*These authors contributed equally to this work.

Received 2 January 2012; revised 26 February 2012; accepted 26

February 2012; published online 30 March 2012 last three decades. It is the sixth leading cancer by incidence worldwide and only $40-50 \%$ of patients with head and neck squamous cell carcinoma will survive for 5 years. ${ }^{1}$ Two-thirds of the diagnosed tumors present with locally advanced or metastatic disease (stages III and IV) and patients survival is determined by the development of loco-regional recurrences, distant metastases and second primary tumors. ${ }^{2}$ In this context, the 
search for novel diagnostic or prognostic markers that leads to improved survival rates for head and neck squamous cell carcinoma becomes essential. ${ }^{3}$

Recent advances in genomic and basic research have increased our understanding of the molecular processes governing tumor formation and progression. Numerous studies have demonstrated the involvement of ion channels in the pathogenesis of various diseases, including cancer. ${ }^{4-7}$ Potassium channels were originally identified in excitable cells, but they are present in virtually all types of cells, where they are involved in a multitude of physiological functions. ${ }^{7-10}$ Because of their oncogenic properties, distribution, modulation and pharmacology, members of the ether à-go-go (EAG) potassium channels family have gained interest as research tools for detection and therapy of different cancers. ${ }^{11-15}$ The EAG potassium channel family comprises three distinct subfamilies: EAG (Kv10), ERG (EAG-related gene; Kv11) and ELK (EAG-like; Kv12). Attempts to clone the human EAG channel led to the detection of the human ERG (HERG) channel. ${ }^{16}$

HERG channel activity plays an important role in cardiac and other excitable cells. The rapidly activating component of the delayed rectifying $\mathrm{K}+$ current $\left(I_{\mathrm{Kr}}\right)$ is mediated by HERG1 in the heart and HERG1 currents blockade may lead to serious ventricular arrhythmias and sudden death. ${ }^{17,18}$ In addition, HERG1 channels are frequently aberrantly expressed in a variety of primary tumors and cancer cell lines of different origin (reviewed in ref.6) where they have been shown to regulate various functions relevant to tumor cell biology, such as cell proliferation, apoptosis, cell migration, invasiveness and neoangiogenesis by modulating several biochemical pathways. ${ }^{13,14}$

To date, no information exists about the possible contribution of HERG1 to the development and progression of head and neck squamous cell carcinoma. This study is the first to investigate the expression pattern of HERG1 in both primary tumors and precancerous lesions to establish the clinical and biological role of HERG1 potassium channel subunit in the initiation and progression of head and neck squamous cell carcinoma.

\section{Materials and methods}

\section{Patients and Tissue Specimens}

Surgical tissue specimens from 75 patients with premalignant lesions of the larynx and 133 patients with laryngeal or hypopharyngeal squamous cell carcinoma who underwent surgical treatment at the Hospital Universitario Central de Asturias between 1996 and 2005 were retrospectively collected, following institutional review board guidelines. Representative tissue sections were obtained from archival, paraffin-embedded blocks and the histolo- gical diagnosis was confirmed by an experienced pathologist (AA). The sections selected for study were as follows: In the group of patients with premalignant lesions, the entire lesion was included in one block and therefore the section used for histological diagnosis was subsequently stained and evaluated. The premalignant lesions were classified following the World Health Organization classification $^{19}$ into the categories of mild dysplasia (10 lesions, 13\%), moderate dysplasia (25 lesions, $33 \%$ ) or severe dysplasia/carcinoma 'in situ' (CIS; 40 lesions, 54\%). All patients were men, with a mean age of 65 years (range 36-83 years). All of them were smokers, and 23 were also habitual alcohol drinkers. Patients with a diagnosis of premalignant lesion and cancer within the next 6 months were excluded from the study. All patients were treated by excisional biopsy using stripping microflap excision. Patients were followed up for a minimum of 60 months or until progression to malignancy occurred.

In the group of patients with head and neck squamous cell carcinoma, three morphologically representative areas were selected from each individual tumor block to construct five tissue microarray blocks. All patients had a single primary tumor, microscopically clear surgical margins and received no treatment before surgery. Only five patients were women, the mean age was 60 years (range 38-86 years). All but three patients were habitual tobacco and alcohol consumers. Of 133 patients, $71(53 \%)$ received postoperative radiotherapy. The characteristics of the patients studied and the clinicopathological features of their tumors (site, pT classification, pN classification, disease stage and histopathological grade) are shown in Table 1 . The stage of disease was determined after the surgical resection of the tumor according to the TNM system of the International Union against Cancer (6th Edition). The histological grade was determined according to the degree of differentiation of the tumor (Broders' classification).

\section{Tissue Microarray Construction}

A series of 133 patients affected by laryngeal/ hypopharyngeal squamous cell carcinomas were selected to construct tissue microarrays, with institutional review board approval, from archived formalin-fixed, paraffin-embedded head and neck squamous cell carcinoma tissue samples (Department of Pathology at the Hospital Universitario Central de Asturias). A high percentage of patients who developed distant metastasis was included (52 of $133,39 \%$ ) to investigate the possible role of HERG1 expression in metastatic dissemination. The original archived hematoxylin- and eosin-stained slides were reviewed by a pathologist (AA) and $1 \mathrm{~mm}$ diameter tissue cores from donor blocks were 
Table 1 Characteristics of the patient population and clinicopathological parameters of their tumors included in the tissue microarrays

\begin{tabular}{lc}
\hline Characteristic & No. of patients (\%) \\
\hline Mean age (years) & 60 \\
Gender & \\
Men & 128 \\
Women & 5 \\
pT classification & \\
T1 & 20 \\
T2 & 21 \\
T3 & 47 \\
T4 & 45 \\
pN classification & \\
N0 & \\
N1 & 44 \\
N2 & 16 \\
N3 & 64 \\
& 9 \\
Disease stage & \\
I & \\
II & \\
III & \\
IV & 54 \\
Pathological grade & 5 \\
Well differentiated & 25 \\
Moderately differentiated & 89 \\
Poorly differentiated & \\
Site & \\
Hypopharynx & 38 \\
Larynx & 59 \\
Total & 36 \\
& \\
& \\
& \\
& \\
&
\end{tabular}

transferred to a recipient 'Master' block in a gridlike manner using a manual tissue microarray instrument. Three tissue cores were taken from the donor tissue blocks to fully represent each case. In addition, each tissue microarray also contained three cores of normal epithelium as an internal control.

To check the histopathological diagnosis and the adequacy of tissue sampling, a section from each microarray was stained with hematoxylin and eosin and examined by light microscopy.

\section{Immunohistochemistry}

The formalin-fixed, paraffin-embedded tissues were cut into $3-\mu \mathrm{m}$ sections and dried on Flex IHC microscope slides (Dako). The sections were deparaffinized with standard xylene and hydrated through graded alcohols into water. Antigen retrieval was performed using Envision Flex Target Retrieval solution, high $\mathrm{pH}$ (Dako). Staining was carried out at room temperature on an automatic staining workstation (Dako Autostainer Plus) with rabbit polyclonal anti-HERG1 (CT) (pan) antibody
(Enzo Life Sciences), at 1:200 dilution using the Dako EnVision Flex + Visualization System (Dako Autostainer). Counterstaining with hematoxylin for $1 \mathrm{~min}$ was the final step.

As HERG1 staining showed a homogeneous distribution, a qualitative scoring system based on staining intensity was applied. Immunostaining was scored blinded to clinical data by two independent observers as negative (0), weakly $(1+)$, moderately $(2+)$ or strongly positive $(3+)$. Scores $\geq 2$ were considered as HERG1-positive expression.

\section{Cell Culture}

The head and neck squamous cell carcinomaderived cell lines SCC2, SCC38, SCC40 and SCC42B were kindly provided by $\mathrm{Dr} \mathrm{R}$ Grenman ${ }^{20}$ and the cell lines SCC040, SCC041, SCC078, SCC094, SCC096A and SCC120 were established by Dr MA Hermsen. ${ }^{19}$ Cells were grown in DMEM supplemented with $10 \%$ fetal bovine serum, $100 \mathrm{U} / \mathrm{ml}$ penicillin, $200 \mu \mathrm{g} / \mathrm{ml}$ streptomycin, $2 \mathrm{mmol} / \mathrm{l}$ L-glutamine, $20 \mathrm{mmol} / \mathrm{l}$ HEPES (pH 7.3) and $100 \mu \mathrm{mol} / \mathrm{l}$ nonessential amino acids.

\section{Real-Time RT-PCR}

Total RNA was extracted using Trizol reagent (Invitrogen Life Technologies) and cDNA was synthesized with Thermoscript RT-PCR System (Invitrogen Life Technologies) according to the manufacturer's protocols. Gene expression was analyzed by Realtime PCR using the ABI PRISM 7500 Sequence detector following Applied Biosystems' SYBR Green Master Mix protocol. Reactions were carried out using primers specific for herg1 forward $5^{\prime}$-AC CGTGAGATCATAGCACCTAA-3' $3^{\prime}$ and reverse $5^{\prime}$ ACAGGACCTGGGTGACCTTCT- ${ }^{\prime}$, and the constitutively expressed L19 ribosomal coding gene as internal control. The relative mRNA expression was calculated using the $2^{-\triangle \Delta C T}$ method and the data were expressed as the fold change in herg1 levels in the squamous cell carcinoma-derived cell lines normalized to L19 levels. Single PCR products of the correct size were observed in all cases.

\section{siRNA Transfections}

siRNA duplexes were purchased from Thermo Scientific Dharmacon (Lafayette, CO, USA). Human KCNH2 ON-TARGETplus SMARTpool was used to knockdown HERG1 expression and SiGENOME Nontargeting siRNA Pool No. 2 was used as a negative control siRNA.

Head and neck squamous carcinoma cells were seeded into 6-well plates in antibiotic-free medium at a density of 60000 cells per well. The next day, cells were transfected with pooled siRNA duplexes 
using Lipofectamine 2000 (Invitrogen) according to the manufacturer's protocol.

\section{Anchorage-Dependent and -Independent Cell Growth}

Head and neck squamous carcinoma-derived cells were seeded into 96-well plates at a density of 1500 cells per well, treated with $10 \mu \mathrm{M}$ imipramine or $5 \mu \mathrm{M}$ E-4031 (Sigma-Aldrich) and cell proliferation was measured at different time points (2, 4 and 6 days). Growth medium containing the corresponding drug was replaced every $48 \mathrm{~h}$. Quantification of cell number was determined in quadruplicates using a tetrazolium-based MTS test (CellTiter 96 AQueous One Solution Cell Proliferation Assay from Promega, Madison, WI, USA) reading the absorbance at $490 \mathrm{~nm}$.

Anchorage-independent cell growth was estimated by using 96-well plates coated with an anti-adhesive polymer poly-(2-hydroxyethyl methacrylate) (polyHEMA; Sigma-Aldrich).

\section{Matrigel Invasion Assays}

In vitro invasion assays were performed by using a 24-well invasion chamber coated with Matrigel (Becton Dickinson). Cells $\left(3 \times 10^{4}\right)$ were seeded in $500 \mu \mathrm{l}$ of serum-free DMEM in the upper chamber of the prehydrated Matrigel-coated inserts and $500 \mu \mathrm{l}$ of DMEM containing $10 \%$ of fetal bovine serum was added to the lower chamber. After $48 \mathrm{~h}$, the cells remaining in the upper surface of the insert were removed with a cotton swab, whereas invaded cells on the underside of the insert were fixed and stained with a solution containing $0.5 \%$ crystal violet (Sigma Aldrich) in methanol. The total number of invading cells was quantified using the Leica QWin Pro V3.2.1 Image Analysis System (Copyright Leica Microsystems, Heerbrugg, Switzerland) on images acquired with an Olympus BX61 Microscope and DP70 Digital Camera System.

\section{Statistical Analyses}

The $\chi^{2}$ test and Fisher's exact test were used for comparison between categorical variables and Student's $t$-test for parametric continuous variables. Spearman's non-parametric correlation coefficient for comparison between HERG1 protein expression in the premalignant lesion and the invasive tumor subsequently developed. For time-to-event analysis, Kaplan-Meier curves were plotted. Differences between survival times were analyzed by the logrank method. Cox proportional hazards models were utilized for univariate and multivariate analyses. The HR with $95 \% \mathrm{CI}$ and $P$-values were reported. All tests were two-sided. The values of $P \leq 0.05$ were considered statistically significant.

\section{Results}

HERG1 Protein Expression in Invasive Head and Neck Squamous Cell Carcinomas

Immunohistochemical analysis of HERG1 expression was carried out on head and neck squamous cell carcinoma tissue microarrays composed of tissue sections from 133 laryngeal/hypopharyngeal squamous cell carcinomas. Each tissue microarray also contained sections of normal epithelium as internal control. Immunostaining was successfully evaluated in $124(93 \%)$ of 133 cases. In all, 108 $(87 \%)$ of the 124 tumors exhibited HERG1 positiveexpression (moderate to strong expression; Figures 1a and b), whereas HERG1 expression was negligible in both normal epithelium and stromal cells (Figure 1c). HERG1 expression in tumors was preferentially detected in the cytoplasm, with protein enrichment at the cell periphery in some cases.

\section{Associations of HERG1 Protein Expression with Clinicopathological Parameters and Patient Outcome}

The relationships between HERG1 protein expression and the clinicopathological variables are shown in Table 2. HERG1 expression was significantly associated with lymph node metastasis (N1-3 versus No, $P=0.04$ ) and advanced disease stages (III and IV versus I-II, $P<0.001)$. The median follow-up of the whole series was 24.5 months (range, 1-97 months), and the median follow-up of the patients alive at the last visit was 67.5 months (range, 48-97 months). In all, 15 patients died for other causes not related to the primary tumor before 36 months of follow-up. These cases were excluded from the recurrence analysis. Of the remaining 109 patients, 9 cases (8\%) developed local recurrence, $10(9 \%)$ regional recurrence and 6 (5\%) loco-regional recurrence. In addition, we included in the tissue microarray 49 cases $(45 \%$ of total cases) who developed distant metastasis (20 of them also had loco-regional recurrence). HERG1 expression correlated significantly with regional tumor recurrence $(P=0.004)$ and distant metastasis $(P=0.03)$, but not with local recurrence $(P=0.07)$. Of note, HERG1-positive expression was found in $16(100 \%)$ of 16 cases with regional recurrences and $47(96 \%)$ of 49 patients who developed distant metastasis. In addition, univariate Kaplan-Meier analysis demonstrated a significant correlation between HERG1-positive expression and reduced disease-specific survival (logrank test, $P=0.012$; Figure 2a). Other variables associated with a poorer prognosis were hypopharyngeal location of the tumor $(P=0.004)$, T4 classification $(P=0.023)$, presence of nodal metastases $(P<0.001)$ and poor histological differentiation $(P=0.005)$. However, in multivariate analysis the only parameters independently associated with a reduced disease-specific survival were the presence of nodal metastasis ( $\mathrm{HR}=3.25$; CI 95\% 1.7-6.2; 

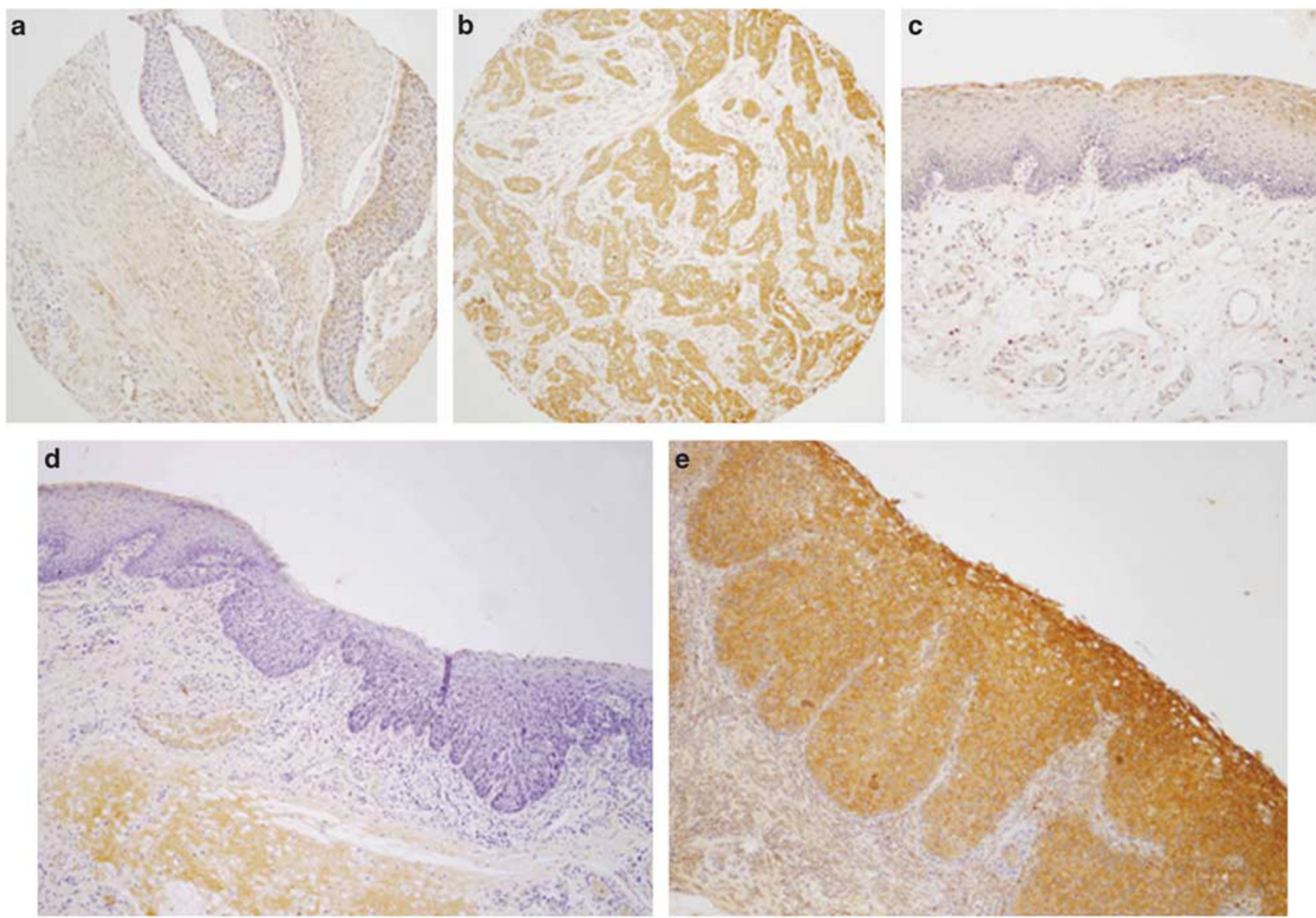

Figure 1 Immunohistochemical analysis of human ether à-go-go-related gene 1 (HERG1) expression. Representative examples of invasive head and neck squamous cell carcinomas showing HERG1-negative staining (a) and strong positive staining (b). Normal-adjacent epithelia showed negative HERG1 staining (c). Representative examples of laryngeal squamous intraepithelial lesions showing areas of severe dysplasia with negative (d) and positive HERG1 staining (e).

$P<0.001)$ and poor histological differentiation $(\mathrm{HR}=1.29$; CI $95 \% 1.08-1.66 ; P=0.043)$.

\section{HERG1 Protein Expression in the Early Stages of Tumorigenesis}

HERG1 protein expression was evaluated by immunohistochemistry on a set of 75 laryngeal premalignancies to determine the timing of HERG1 expression during head and neck squamous cell carcinoma tumorigenesis. Of the 75 laryngeal lesions displayed, 31 (41\%) HERG1-positive expression in the dysplastic areas with negligible immunostaining in both stromal cells and normaladjacent epithelia (Figures 1d and e). In all, 2 (20\%) of the 10 lesions with mild dysplasia, $10(40 \%)$ of the 25 lesions with moderate dysplasia and 19 $(47 \%)$ of the 40 lesions with severe dysplasia/CIS exhibited HERG1-positive staining $(P=0.28)$.

The evolution to invasive carcinoma in relation to the histopathological diagnosis and HERG1 protein expression is shown in Table 3. During the followup period, $25(33 \%)$ of 75 patients developed an invasive carcinoma at the same site of the previous premalignant lesion. The mean time to cancer diagnosis in the cases that progressed was 29 months (range 12-66 months). In this cohort study, there was no statistically significant correlation between the histopathological grade and the risk of progression to laryngeal cancer $(P=0.193$; Table 3$)$, although severe dysplasias showed a higher cancer risk $(\mathrm{HR}=1.5 ; 95 \%$ CI: $0.77-2.89 ; P=0.22$, Cox regression).

Interestingly, patients with HERG1-positive lesions had a significantly higher laryngeal cancer incidence than did those with negative lesions (Figure 2b; $\mathrm{HR}=1.69 ; \quad 95 \% \quad \mathrm{CI}$ : 1.12-2.55; $P=0.012$, Cox regression). At 5 years after the patients were diagnosed, $16(52 \%)$ of the 31 patients with positive HERG1 expression developed laryngeal cancer, whereas only $9(20 \%)$ of the 44 patients with negative expression progressed to invasive carcinoma $(P=0.007$; Table 3$)$.

HERG1 protein expression was also determined in 19 of the 25 invasive tumors developed in our cohort. For each patient, protein expression in the invasive tumor was compared with that of the 
Table 2 Associations between HERG1 protein expression and clinicopathological findings, site, relapse and disease outcome

\begin{tabular}{|c|c|c|c|}
\hline Characteristic & No. & $\begin{array}{l}\text { HERG1 protein } \\
\text { expression (\%) }\end{array}$ & $\begin{array}{c}\mathrm{P}- \\
\text { value }\end{array}$ \\
\hline \multicolumn{4}{|l|}{$p T$ classification } \\
\hline $\mathrm{T} 1-\mathrm{T} 2$ & 35 & $28(80)$ & \multirow[t]{3}{*}{$0.22^{\mathrm{a}}$} \\
\hline T3 & 45 & $39(87)$ & \\
\hline $\mathrm{T} 4$ & 44 & $41(93)$ & \\
\hline \multicolumn{4}{|l|}{$p N$ classification } \\
\hline No & 39 & $30(77)$ & \multirow[t]{2}{*}{$0.04^{\mathrm{b}}$} \\
\hline N1-3 & 85 & $78(92)$ & \\
\hline \multicolumn{4}{|l|}{ Disease stage } \\
\hline I-II & 15 & $8(53)$ & \multirow[t]{3}{*}{$0.000^{\mathrm{a}}$} \\
\hline III & 24 & $22(92)$ & \\
\hline IV & 85 & $78(92)$ & \\
\hline \multicolumn{4}{|l|}{ Pathological grade } \\
\hline Well differentiated & 35 & $30(86)$ & \multirow[t]{3}{*}{$0.748^{\mathrm{a}}$} \\
\hline Moderately differentiated & 56 & $48(86)$ & \\
\hline Poorly differentiated & 33 & $30(91)$ & \\
\hline \multicolumn{4}{|l|}{ Site } \\
\hline Hypopharynx & 62 & $56(90)$ & \multirow[t]{2}{*}{$0.422^{\mathrm{b}}$} \\
\hline Larynx & 62 & $52(84)$ & \\
\hline \multicolumn{4}{|l|}{ Recurrence $^{\mathrm{c}}$} \\
\hline No recurrence & 35 & $27(77)$ & \multirow[t]{6}{*}{$0.019^{\mathrm{a}}$} \\
\hline Local recurrence & 9 & $6(66)$ & \\
\hline Regional recurrence & 10 & $10(100)$ & \\
\hline Loco-regional recurrence & 6 & $6(100)$ & \\
\hline $\begin{array}{l}\text { Loco-regional recurrence and } \\
\text { distant metastasis }\end{array}$ & 20 & $20(100)$ & \\
\hline Distant metastasis & 29 & $27(93)$ & \\
\hline \multicolumn{4}{|l|}{ Disease status } \\
\hline Alive without disease & 28 & $20(71)$ & \multirow[t]{3}{*}{$0.004^{\mathrm{b}}$} \\
\hline Dead of index cancer & 70 & $66(94)$ & \\
\hline Died of other causes & 11 & $10(91)$ & \\
\hline Total cases & 124 & $108(87)$ & \\
\hline
\end{tabular}

${ }^{\mathrm{a}} \chi^{2}$ Test.

${ }^{\mathrm{b}}$ Fisher's exact test.

${ }^{\mathrm{C}}$ In all, 15 patients who died from causes not related to the index tumor before 36 months of follow-up were excluded from the recurrence analysis.

corresponding previous premalignant lesion. HERG1-positive expression was detected in 15 $(79 \%)$ of the 19 invasive tumors. Statistical analysis revealed a strong positive correlation between HERG1 expression in patient-matched premalignant lesions versus invasive tumors (Spearman's correlation coefficient 0.489; $P=0.034$ ). Overall, we observed that HERG1 expression was maintained or further augmented in the tumor compared with the patient-matched preinvasive lesion.

\section{In vitro Functional Role of HERG1 Expression in Head and Neck Squamous Cell Carcinoma-Derived Cell Lines}

To assess the pathobiological role of aberrant HERG1 expression in head and neck squamous cell
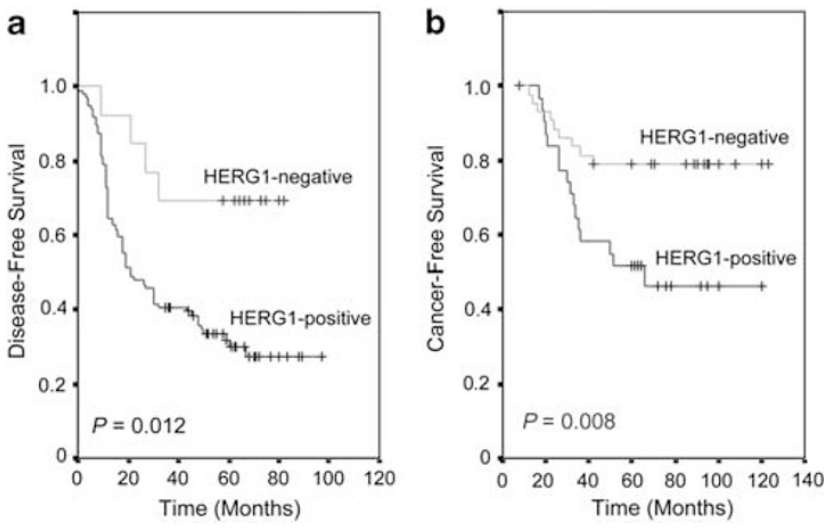

Figure 2 Kaplan-Meier cancer-free survival curves categorized by human ether à-go-go-related gene 1 (HERG1) expression (positive versus negative) in patients with head and neck squamous cell carcinomas (a) and in patients with laryngeal dysplasias (b). $P$-values were estimated using the log-rank test.

carcinoma a series of functional studies using in vitro cellular models were carried out. Thus, we first compared the expression levels of herg1 by realtime RT-PCR in a panel of 10 head and neck squamous cell carcinoma-derived cell lines (Figure 3a). herg1 expression was detected in all squamous cell carcinoma cell lines, although mRNA levels varied depending on the cell line and SCC42B cells showed the highest expression.

We next determined the impact of HERG1 blockade on cell proliferation by using the EAG family blocker imipramine and the HERG1-specific blocker E-4031. We found that the selective blockade of HERG1 ion-conducting function (canonical function) with either imipramine or E-4031 had no effect on the growth of SCC42B cells as monitored by MTS (Figure 3b). However, the inhibition of HERG1 expression by siRNA transfection in SCC42B cells specifically and significantly reduced both the anchorage-dependent and -independent cell growth compared with siControl-transfected cells (Figure 3c). Furthermore, Matrigel invasion assays showed that the invasive capacity of SCC42B cells was dramatically impaired by siRNA-mediated HERG1 knockdown (Figure 3d).

\section{Discussion}

Like other epithelial cancers, head and neck carcinogenesis appears to evolve through a multistep process involving biomolecular changes caused by carcinogen exposure, ensuing premalignant lesions and consequent invasive cancer. ${ }^{1,21}$ The identification of molecular alterations associated with these events could yield insight into the mechanisms of head and neck squamous cell carcinoma initiation and progression and may provide new tools for diagnosis, treatment and prevention. ${ }^{2,3}$

In recent years, the expression and activity of different ion channels have been shown to regulate specific stages of cancer development and 
Table 3 Evolution of the premalignant lesions in relation to the histopathological diagnosis and HERG1 protein expression

\begin{tabular}{lcrl}
\hline Characteristic & No. of cases (\%) & $\begin{array}{c}\text { Progression to } \\
\text { carcinoma (\%) }\end{array}$ & \\
\hline $\begin{array}{l}\text { Histopathological diagnosis } \\
\text { Mild dysplasia }\end{array}$ & $10(13)$ & $2(20)$ & $0.193^{\mathrm{a}}$ \\
$\begin{array}{l}\text { Moderate dysplasia } \\
\text { Severe dysplasia }\end{array}$ & $25(33)$ & $6(24)$ & \\
HERG1 expression & $40(54)$ & $17(42)$ & \\
$\quad$ & & & \\
Negative & $44(59)$ & $9(20)$ & $0.007^{\mathrm{b}}$ \\
Positive & $31(41)$ & $16(52)$ & \\
\hline
\end{tabular}

${ }^{\mathrm{a}} \chi^{2}$ Test.

${ }^{\mathrm{b}}$ Fisher's exact test.

progression. ${ }^{5-7}$ The contribution of potassium channels to the neoplastic phenotype involves, between others, the control of cell proliferation and apoptosis, or the regulation of invasiveness and metastatic spread. ${ }^{9-13,22}$ Owing to their oncogenic properties and location at the cell membrane, members of the EAG channels family have been postulated as promising therapeutic targets for cancer treatment. ${ }^{11,15}$

This study is the first to investigate the expression pattern of the HERG1 potassium channel subunit in laryngeal premalignant lesions and invasive carcinomas to ascertain the role of HERG1 in both malignant transformation and tumor progression and its clinical significance in head and neck squamous cell carcinoma. Our findings demonstrate that HERG1 is frequently aberrantly expressed in an extraordinarily high percentage of primary tumors $(87 \%)$ and head and neck squamous cell carcinoma-derived cell lines $(100 \%)$, whereas no expression was detected in the corresponding normal epithelia, rendering HERG1 expression a reliable tumor marker. HERG1 expression increased during head and neck squamous cell carcinoma progression and was significantly associated with lymph node metastasis, advanced disease stages, regional tumor recurrence, distant metastasis and reduced disease-specific survival. Similarly, HERG1 has also been found frequently aberrantly expressed in other types of cancer, such as endometrial cancer, ${ }^{23}$ leukemia, ${ }^{24}$ glioblastoma, ${ }^{25}$ gastric cancer, ${ }^{26}$ esophageal squamous cell carcinoma $^{27}$ and colorectal cancer, ${ }^{28}$ where HERG1 expression commonly correlated with tumor aggressiveness, poor prognosis and metastatic disease.

Even though cumulative evidences have clearly demonstrated the involvement of HERG1 in tumor progression and metastatic spread, its contribution to malignant transformation has not yet been established. In an attempt to accomplish this, we have investigated the role of HERG1 in the early stages of tumorigenesis by analyzing the expression pattern on a large series of laryngeal precancerous lesions and the tumors subsequently developed. We found that HERG1 expression is a frequent and early event in laryngeal tumorigenesis. HERG1-positive expression was detected in 31 (41\%) of 75 laryngeal dysplasias and was maintained or even augmented in the corresponding patient-matched invasive tumors subsequently developed. Interestingly, patients carrying HERG1-positive lesions experienced a significantly higher cancer incidence than those with negative expression. Although patients with severe dysplasia were at a higher risk of malignant progression, histology did not show a significant role in assessing laryngeal cancer risk in this cohort, which highlights the limited value of histopathological classification in predicting outcome. These data support a novel role for HERG1 in laryngeal cancer development and malignant transformation and, more importantly, uncover the potential clinical utility of HERG1 expression as a biomarker for cancer risk assessment providing additional value beyond current clinical and histopathological criteria. These findings will require further confirmation in large prospective studies before implementation into clinical practice.

HERG1 expression has also been detected in Barrett's esophagus, a preneoplastic condition strongly associated with esophageal adenocarcinoma, and it has been hypothesized that this expression marks an early step of progression from normality to esophageal cancer. ${ }^{29}$ Nevertheless, it remains to be determined whether aberrant HERG1 expression plays an active role in malignant transformation or it is a mere consequence of tumor establishment and progression.

The in vitro functional studies in head and neck squamous cell carcinoma-derived cell lines have further contributed to clarify the pathobiological role of HERG1 potassium channel subunit, demonstrating that HERG1 expression is a biologically relevant feature that promotes cell proliferation and invasiveness. Moreover, the role of HERG1 on cell proliferation seems to be independent of its ionconducting properties, observations in line with those made in breast ${ }^{30}$ and lung ${ }^{31}$ cancer cells. Therefore, consistent with our in vivo findings, aberrant HERG1 expression may confer a proliferative advantage to tumor cells and the acquisition of a truly invasive and metastatic potential, thus favoring tumors to grow faster and with increased aggressiveness, ultimately leading to disease progression. Despite the primary function of voltagedependent ion channels is to regulate $\mathrm{K}+$ flux through the cell membrane, recent studies have shown that HERG1 channels may exert pleiotropic effects in cancer cells by triggering and modulating intracellular signaling cascades through the formation of macromolecular complexes with membrane receptors, especially integrins. ${ }^{14}$ On the basis of current evidence, it has been hypothesized that the activity of HERG1 channels inside these complexes modulates the function of the partner proteins mainly via conformational coupling, independently of the ion-conducting function. In addition, it has been proposed that the HERG1-centered plasma 

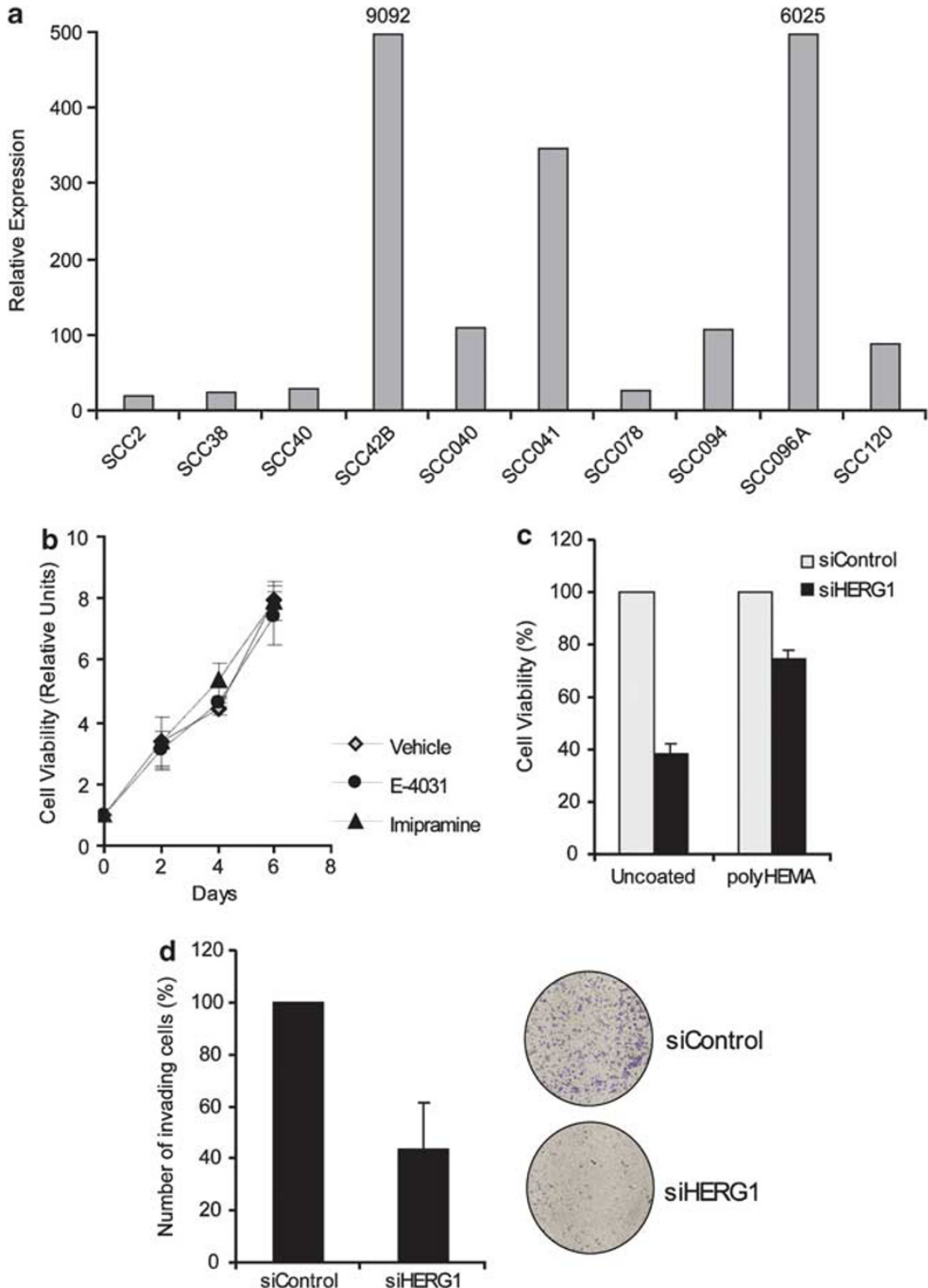

Figure 3 Effect of human ether à-go-go-related gene 1 (HERG1) blockade on the growth and invasion of head and neck squamous cell carcinoma-derived cells. (a) Comparison of herg1 levels by real-time reverse transcription-PCR (RT-PCR) in head and neck squamous cell carcinoma-derived cell lines. (b) Tetrazolium-based MTS (3-(4,5-dimethylthiazol-2-yl)-5-(3-carboxymethoxyphenyl)-2-(4-sulfophenyl)$2 \mathrm{H}$-tetrazolium) proliferation assay in SCC42B cells treated with $10 \mu \mathrm{M}$ imipramine or $5 \mu \mathrm{M}$ E-4031 for 2, 4 and 6 days. Data are expressed relative to the absorbance at day 0 of vehicle-treated cells. The results shown represent the average of two independent experiments assayed in quadruplicate \pm s.d. (c) SCC42B cells were transfected with the indicated short interfering RNAs (siRNAs) and the anchoragedependent and -independent growth was measured by MTS after 4 days using, respectively, uncoated or poly-2-hydroxyethyl methacrylate (poly-HEMA)-coated tissue culture plastic 96-well plates. Data are expressed as the percentage of viable cells (absorbance at day 4 relative to day 0 of siControl-transfected cells). (d) Matrigel invasion assays were performed in SCC42B cells $72 \mathrm{~h}$ after transfection with either siHERG1 or siControl. The invading cells were stained with crystal violet and quantified using the Leica QWin Pro Image Analysis System.

membrane complexes, being specific to cancer cells, could represent novel targets for antineoplastic therapy.

Pharmacological blockade of HERG1 channels with specific blockers has proved to be effective in significantly reducing the growth of different cancer cells. ${ }^{6,28,32,33}$ In contrast, increasing data indicate that the ion-conducting function of HERG1 may not be a major determinant for its oncogenic potential. ${ }^{14,30,31}$ Furthermore, pharmacological blockade of HERG1 may also have limited therapeutic applicability in human cancers owing to its cardio toxic side effects. ${ }^{34}$ It has recently been described that $R$-roscovitine, a cyclin-dependent kinase inhibitor currently undergoing phase II clinical trials as an anticancer drug, blocks HERG potassium currents at clinically relevant concentrations in HEK293 cells stably expressing HERG 
without significantly increasing the risk of cardiac arrhythmias. $^{35}$ Plasmid vector-mediated HERG1 RNA interference is an alternative approach that has been used to successfully inhibit tumor growth in nude mice. ${ }^{36}$

Collectively, these findings demonstrate that HERG1 potassium channel subunit is frequently aberrantly expressed in an overwhelming percentage of primary tumors and head and neck squamous cell carcinoma-derived cell lines and represents a clinically and biologically relevant feature during disease progression and a promising candidate as tumor marker and membrane therapeutic target for head and neck squamous cell carcinoma treatment. In addition, this study also provides original evidence supporting the involvement of HERG1 in malignant transformation and its potential clinical utility as a novel biomarker for cancer risk assessment, together with morphological criteria.

\section{Acknowledgements}

We thank Pablo Martínez-Camblor (Oficina de Investigación Biosanitaria, OIB) for his assistance with statistical analyses and Teresa Ortega and OIB staff for the administrative support. This work was supported by grants from Fondo de Investigación Sanitaria CP07/00032 and PI10/00157 (to JMGP), PI11/00929 (to CS), ISCIII Fondos FEDER, RTICC (RD06/0020/0034) and Obra Social Cajastur-IUOPA. STM and SAT are recipients of a fellowship from FICYT (BP08-007 and BP11-114).

\section{Disclosure/conflict of interest}

The authors declare no conflict of interest.

\section{References}

1 Forastiere A, Koch W, Trotti A, et al. Head-and-neck cancer. N Engl J Med 2001;345:1890-1900.

2 Haddad RI, Shin DM. Recent advances in head and neck cancer. N Engl J Med 2008;359:1143-1154.

3 Leemans CR, Braakhuis BJ, Brakenhoff RH. The molecular biology of head and neck cancer. Nat Rev Cancer 2011;11:9-22.

4 Kass RS. The channelopathies: novel insights into molecular and genetic mechanisms of human disease. J Clin Invest 2005;115:1986-1989.

5 Wulff H, Castle NA, Pardo LA. Voltage-gated potassium channels as therapeutic targets. Nat Rev Drug Discov 2009;8:982-1001.

6 Arcangeli A, Crociani O, Lastraioli E, et al. Targeting ion channels in cancer: a novel frontier in antineoplastic therapy. Curr Med Chem 2009;16:66-93.

7 Niemeyer BA, Mery L, Zawar C, et al. Ion channels in health and disease. EMBO Rep 2001;2:568-573.

8 O’Grady SM, Lee SY. Molecular diversity and function of voltage-gated $(\mathrm{Kv})$ potassium channels in epithelial cells. Int J Biochem Cell Biol 2005;37:1578-1594.
9 Kaczmarek LK. Non-conducting functions of voltagegated ion channels. Nat Rev Neurosci 2006;7:761-771.

10 Wang Z. Roles of $\mathrm{K}+$ channels in regulating tumour cell proliferation and apoptosis. Pflugers Arch 2004;448:274-286.

11 Camacho J. Ether à go-go potassium channels and cancer. Cancer Lett 2006;233:1-9.

12 Pardo LA, del Camino D, Sanchez A, et al. Oncogenic potential of EAG K+ channels. EMBO J 1999;18:55405547.

13 Arcangeli A. Expression and role of hERG channels in cancer cells. Novartis Found Symp 2005;266:225-232.

14 Pillozzi S, Arcangeli A. Physical and functional interaction between integrins and hERG1 channels in cancer cells. Adv Exp Med Biol 2010;674:55-67.

15 Asher V, Sowter H, Shaw R, et al. Eag and HERG potassium channels as novel therapeutic targets in cancer. World J Surg Oncol 2010;8:113.

16 Warmke JW, Ganetzky B. A family of potassium channel genes related to eag in Drosophila and mammals. Proc Natl Acad Sci USA 1994;91:3438-3442.

17 Trudeau MC, Warmke JW, Ganetzky B, et al. HERG, a human inward rectifier in the voltage-gated potassium channel family. Science 1995;269:92-95.

18 Sanguinetti MC, Jiang C, Curran ME, et al. A mechanistic link between an inherited and an acquired cardiac arrhythmia: HERG encodes the Ipotassium channel. Cell 1995;81:299-307.

19 Hermsen MA, Joenje H, Arwert F, et al. Centromeric breakage as a major cause of cytogenetic abnormalities in oral squamous cell carcinoma. Genes Chromosomes Cancer 1996;15:1-9.

20 Lansford CD, Grénman R, Bier $\mathrm{H}$, et al. Head and neck cancers. In: Masters JRW, Plasson B (eds). Human Cell Culture. Kluwer Academic Press: Dordrecht, 1999, pp $185-255$

21 Califano J, van der Riet P, Westra W, et al. Genetic progression model for head and neck cancer: implications for field cancerization. Cancer Res 1996;56:24882492.

22 Jehle J, Schweizer PA, Katus HA, et al. Novel roles for hERG K(+) channels in cell proliferation and apoptosis. Cell Death Dis 2011;2:e93.

23 Cherubini A, Taddei GL, Crociani O, et al. HERG potassium channels are more frequently expressed in human endometrial cancer as compared to noncancerous endometrium. Br J Cancer 2000;83:17221729.

24 Pillozzi S, Brizzi MF, Bernabei PA, et al. VEGFR-1 (FLT-1), beta1 integrin, and hERG $\mathrm{K}+$ channel for a macromolecular signaling complex in acute myeloid leukemia: role in cell migration and clinical outcome. Blood 2007;110:1238-1250.

25 Masi A, Becchetti A, Restano-Cassulini R, et al. hERG1 channels are overexpressed in glioblastoma multiforme and modulate VEGF secretion in glioblastoma cell lines. Br J Cancer 2005;93:781-792.

26 Ding XW, Yang WB, Gao S, et al. Prognostic significance of hERG1 expression in gastric cancer. Dig Dis Sci 2010;55:1004-1010.

27 Ding XW, Luo HS, Luo B, et al. Overexpression of hERG1 in resected esophageal squamous cell carcinomas: a marker for poor prognosis. J Surg Oncol 2008;97:57-62.

28 Lastraioli E, Guasti L, Crociani O, et al. herg1 gene and HERG1 protein are overexpressed in colorectal cancers and regulate cell invasion of tumor cells. Cancer Res 2004;64:606-611. 
29 Lastraioli E, Taddei A, Messerini L, et al. hERG1 channels in human esophagus: evidence for their aberrant expression in the malignant progression of Barrett's esophagus. J Cell Physiol 2006;209: 398-404.

30 Roy J, Vantol B, Cowley EA, et al. Pharmacological separation of hEAG and hERG $\mathrm{K}+$ channel function in the human mammary carcinoma cell line MCF-7. Oncol Rep 2008;19:1511-1516.

31 Glassmeier G, Hempel K, Wulfsen I, et al. Inhibition of HERG1 $\mathrm{K}(+)$ channel protein expression decreases cell proliferation of human small cell lung cancer cells. Pflugers Arch 2012;463:365-376.

32 Pillozzi S, Brizzi MF, Balzi M, et al. HERG potassium channels are constitutively expressed in primary human acute myeloid leukemias and regulate cell proliferation of normal and leukemic hemopoietic progenitors. Leukemia 2002;16:1791-1798.

33 Shao XD, Wu KC, Hao ZM, et al. The potent inhibitory effects of cisapride, a specific blocker for human ethera-go-go-related gene (HERG) channel, on gastric cancer cells. Cancer Biol Ther 2005;4:295-301.

34 Sanguinetti MC, Tristani-Firouzi M. hERG potassium channels and cardiac arrhythmia. Nature 2006;440: 463-469.

35 Ganapathi SB, Kester M, Elmslie KS. State-dependent block of HERG potassium channels by $R$-roscovitine: implications for cancer therapy. Am J Physiol Cell Physiol 2009;296:C701-C710.

36 Zhao J, Wei XL, Jia YS, et al. Silencing of herg gene by shRNA inhibits SH-SY5Y cell growth in vitro and in vivo. Eur J Pharmacol 2008;579:50-57. 